\title{
Adenoid dysplasia of the oral mucosa
}

\author{
Belinda Bunn, BDS, FC Path(SA) Oral Path, MDent, ${ }^{\mathrm{a}}$ Keith Hunter, BSc (Hons), BDS (Hons), FDS RCSEd, PhD, FRCPath, ${ }^{\text {a,b }}$

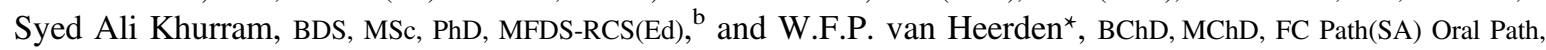 \\ Ph.D, DSC
}

\begin{abstract}
Objective. To describe an unusual variant of oral epithelial dysplasia and to provide an appraisal of its immunohistochemical profile.

Study Design. An unusual form of epithelial dysplasia, which we have termed adenoid dysplasia, was evaluated for staining of cytokeratins AE1/AE3, vimentin, E-cadherin, and $\beta$-catenin. The immunohistochemical results were compared with those observed in moderate epithelial dysplasia, moderately differentiated squamous cell carcinoma, and acantholytic squamous cell carcinoma.

Results. The immunoprofile of adenoid dysplasia was similar to that of acantholytic squamous cell carcinoma. Cytokeratin positivity within the acantholytic dysplastic cells confirmed their epithelial nature, and upregulation of vimentin was suggestive of epithelial-mesenchymal transition. The most distinctive finding was a loss of E-cadherin expression within the discohesive cells, accompanied by increased cytosolic expression of $\beta$-catenin.

Conclusions. This report presents the histomorphologic features of a unique form of oral epithelial dysplasia, termed adenoid dysplasia.
\end{abstract}

The burden of head and neck cancer remains a global public health problem despite advances in diagnostic techniques and therapeutic management. Squamous cell carcinoma is the most predominant epithelial malignancy in this anatomic region, with up to half of all newly diagnosed cases that involve the oral cavity and lip having a strong association with tobacco use and alcohol consumption. ${ }^{1}$ The poor survival in patients with oral cancer is often due to late presentation in patients with advanced-stage disease. The early detec-tion and identification of patients at risk is thus essential for preventative management. At a microscopic level, the development of invasive carcinoma is often pre-ceded by oral epithelial dysplasia. ${ }^{2}$ At present, histo-logic grading of oral epithelial dysplasia remains most predictive of lesion progression to invasive malignancy. ${ }^{1,2}$ The World Health Organization grades dysplasia as mild, moderate, or severe, with full-thickness epithelial changes being referred to as carcinoma in situ. $^{3}$ The subjective nature of this grading system has led to alternative proposals for modification to the classification, including the use of a binary system that categorizes lesions as low-risk or high-risk. ${ }^{4}$

\footnotetext{
${ }^{a}$ Department of Oral Pathology and Oral Biology, School of Dentistry, Faculty of Health Sciences, University of Pretoria. bepartment of Oral and Maxillofacial Pathology, University of Sheffield.

${ }^{\star}$ W.F.P. van Heerden, Department of Oral Pathology and Oral Biology University of Pretoria, PO Box 1266, Pretoria, South Africa, Willie.vanheerden@up.ac.za
}

We have recently encountered an unusual form of moderate (high-risk) oral epithelial dysplasia in our routine diagnostic histopathology service (University of Pretoria, South Africa). The patient in this case presented with clinical features typical of proliferative verrucous leukoplakia (PVL). The initial incisional biopsy showed features of high-risk epithelial dysplasia in which conspicuous pseudoglandular spaces containing acantholytic dysplastic cells were identified, which we have termed adenoid dysplasia (AD). A second biopsy, taken 2 years later, showed a verrucous carcinoma with the same peculiar glandular spaces as noted before. The aim of this report is to document this unconventional form of oral epithelial dysplasia in a patient with PVL, which to the best of our knowledge has never been described before.

\section{CASE DESCRIPTION AND METHODOLOGY A}

90-year-old woman presented to the clinic in 2009 with a 9-month history of multifocal white oral mucosal lesions that had increased in size according to the history. A clinical diagnosis of PVL was made (Figure 1). The patient had no history of tobacco use or excessive alcohol consumption, and no additional risk factors for the development of oral malignancy could be identified. An incisional biopsy from the right buccal mucosa

\footnotetext{
This work was presented as a poster presentation by the same authors at the 25th European Congress of Pathology, Lisbon, Portugal, August 31 to September 4, 2013.

Supported by a grant from the Cancer Association of South Africa.
} 


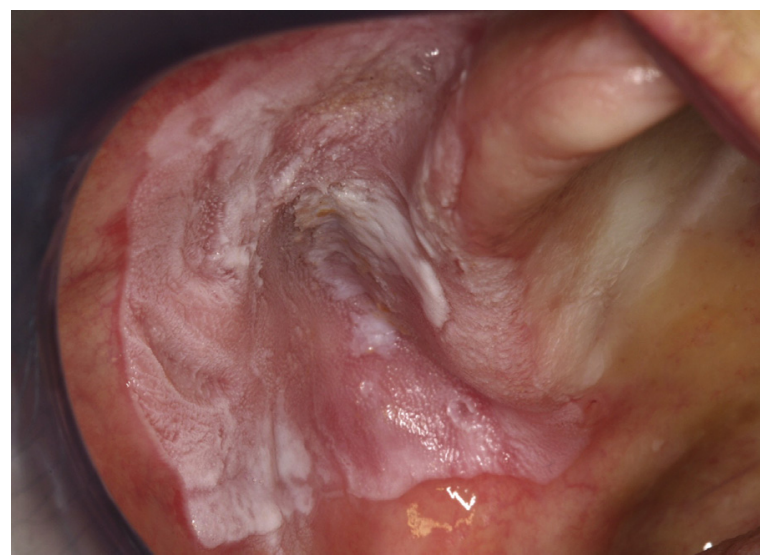

Fig. 1. Clinical features of the lesion on the right buccal vestibule from which the incisional biopsy microscopically designated as adenoid dysplasia was obtained.

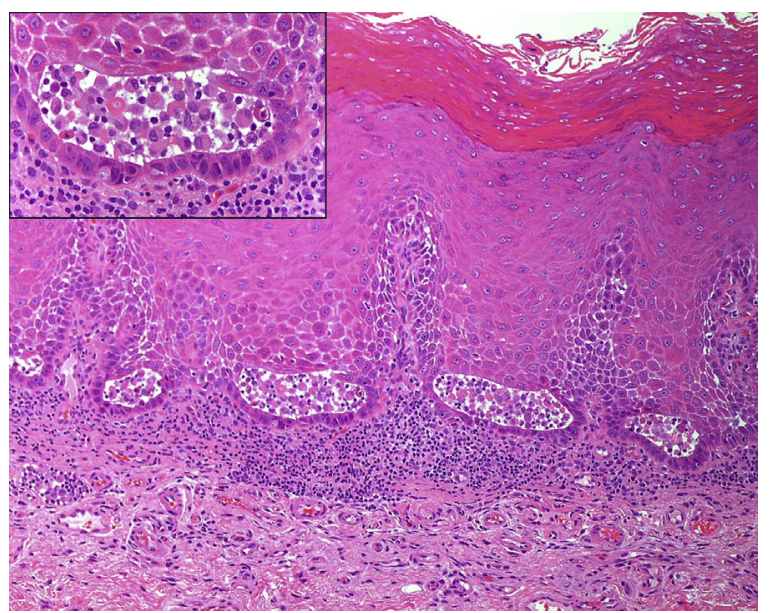

Fig. 2. Histopathologic features of adenoid dysplasia, characterized by dysplastic epithelium in which pseudoglandular spaces are identified, containing free-floating dyskeratotic epithelial cells (hematoxylin-eosin, original magnification $\times 100$; inset, original magnification $\times 400$ ). A high-resolution version of this slide is available as eSlide: VM00349.

showed marked hyperkeratosis and cytologic features in keeping with moderate (high-risk) epithelial dysplasia. Within the basal third of the epithelium were pseudoglandular spaces lined in part by a single layer of cuboidal epithelial cells. Within the central luminal spaces were several acantholytic, dysplastic squamous epithelial cells (Figure 2). These features were distinctly reminiscent of those seen in acantholytic squamous cell carcinoma (ASCC). A patchy interface mucositis was also present. There was no evidence of invasive malignancy in any of the sections examined.

The patient was counseled but refused any treatment. The patient was seen at regular intervals and subsequently developed mixed red and white areas that were

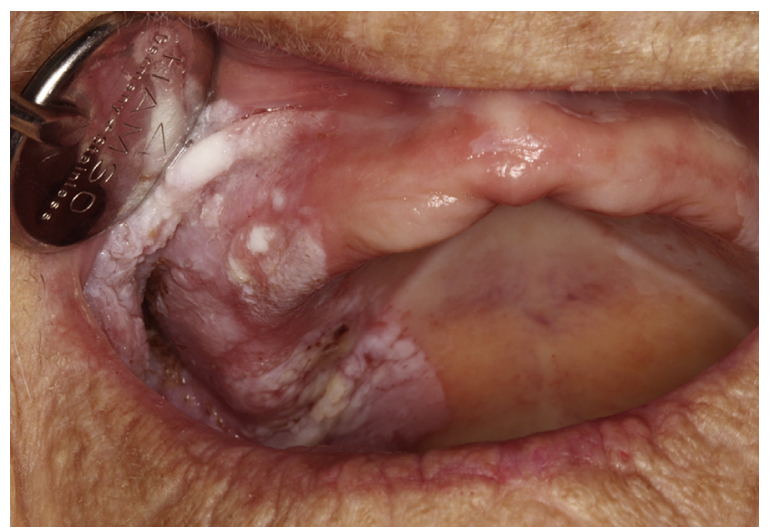

Fig. 3. Follow-up clinical photograph from the same patient 2 years later. A second incisional biopsy, this time obtained from the edentulous maxillary ridge, showed evidence of a verrucous carcinoma.

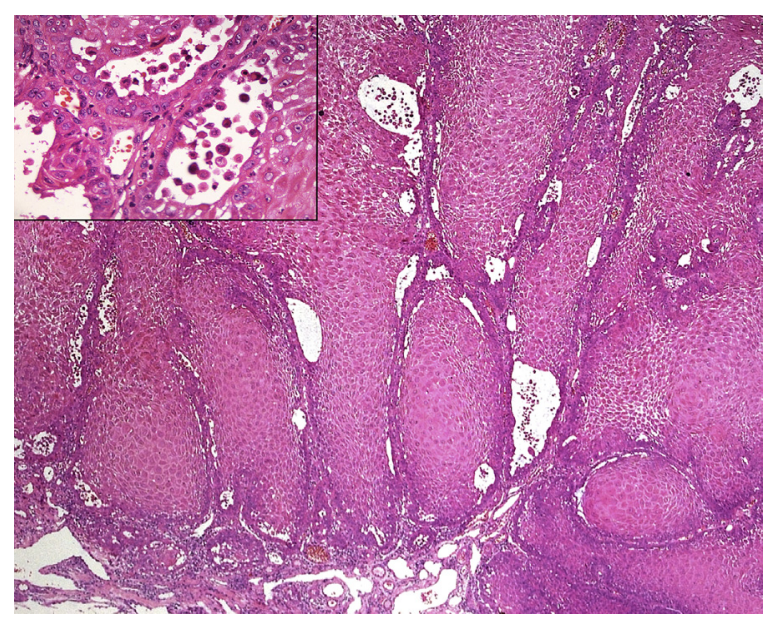

Fig. 4. The histopathologic features noted in the biopsy specimen obtained from the edentulous right maxillary alveolar ridge in the same patient 2 years later. A verrucous carcinoma was diagnosed. The same peculiar adenoid structures identified in the initial dysplastic lesion are represented again (hematoxylin-eosin, original magnification $\times 40$; inset; original magnification $\times 400$ ). A high-resolution version of this slide is available as eSlide: VM00361.

highly suggestive of malignancy (Figure 3). A second biopsy obtained from the upper right edentulous maxillary alveolar ridge in 2011 showed features of a verrucous carcinoma. The very same features of pseudoglandular spaces/adenoid structures containing acantholytic cells were identified within this follow-up biopsy (Figure 4).

The formalin-fixed, paraffin embedded tissue sections were stained for routine histomorphologic assessment by means of hematoxylin-eosin stain. Immunohistochemical staining was performed on $4-\mu \mathrm{m}$ sections, which were first deparaffinized and hydrated and then processed with antigen retrieval. Antibodies to 
Table I. Summary of antibodies used in immunohistochemical assessment

\begin{tabular}{llllll}
\hline Immunohistochemical stain & Antibody & \multicolumn{1}{c}{ Clone } & Dilution & Epitope retrieval & Detection kit \\
\hline AE1/AE3 & Dako IR053 & AE1/AE3 & RTU & Enzyme & Dako Envision + \\
Vimentin & Dako IR630 & V9 & RTU & Low pH HIER & Dako Envision + \\
E-cadherin & Dako IR059 & NCH-38 & RTU & High pH HIER & Novocastra Novolink \\
B-catenin & Dako IR702 & Beta-catenin-1 & RTU & High pH HIER & Novocastra Novolink \\
\hline
\end{tabular}

$H I E R$, heat-induced epitope retrieval; $R T U$, ready to use.

Table II. Immunohistochemical staining in adenoid dysplasia from the initial biopsy and the adenoid structures in the second biopsy compared with the immunoprofiles noted in ASCC, MDSCC, and MED

\begin{tabular}{|c|c|c|c|c|}
\hline Diagnosis & $A E 1 / A E 3$ & Vimentin & E-cadherin & B-catenin \\
\hline $\mathrm{AD}$ & + & Faint to strong cytoplasmic + & - & + cytoplasmic staining \\
\hline Verrucous carcinoma with adenoid features & + & + & - & + cytoplasmic staining \\
\hline ASCC & + & Faint to strong cytoplasmic + & - & + cytoplasmic staining \\
\hline MDSCC & + & - & + & - \\
\hline MED & + & - & + & - \\
\hline
\end{tabular}

AE1/AE3, multicytokeratin AE1, AE3; AD, adenoid dysplasia; $A S C C$, acantholytic squamous cell carcinoma; $M D S C C$, moderately differentiated keratinizing squamous cell carcinoma; $M E D$, moderate epithelial dysplasia.

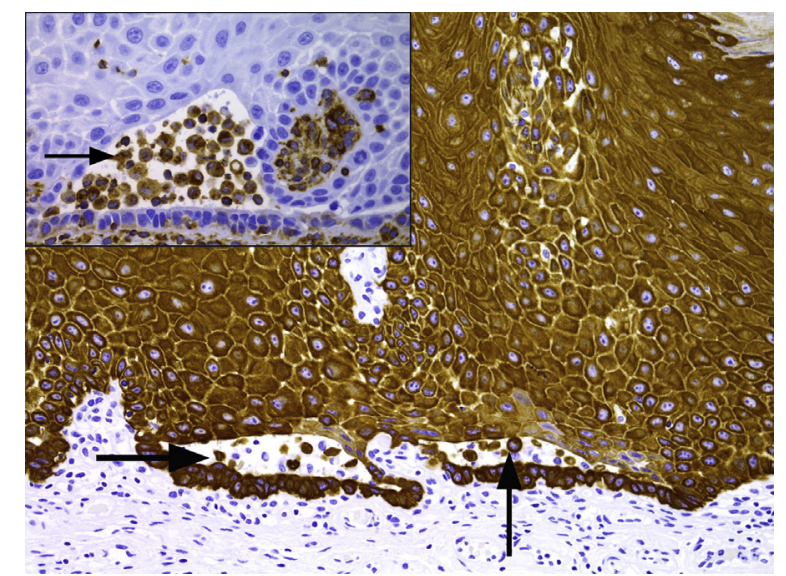

Fig. 5. Cytokeratin positivity confirms the epithelial nature of the free-lying, dysplastic acantholytic cells within the pseudoglandular spaces of the initial biopsy of adenoid dysplasia (AE1/AE3, original magnification $\times 400$ ). The inset shows coexpression of vimentin by the same cells (vimentin, original magnification $\times 400$ ). High-resolution versions of the slide images are available as eSlide: VM00350, eSlide: VM00351.

cytokeratins AE1/AE3, vimentin, E-cadherin, and $\beta$ catenin were obtained from Dako (Table I). Each antibody was supplied prediluted and ready to use in liquid form within a buffer containing stabilizing protein and $0.015 \mathrm{~mol} / \mathrm{L} \mathrm{NaN}_{3}$. The antibody concentrations supplied were optimized for use on Dako's autostainer. Positive and negative controls were used for verification of the staining in each case. The immunostaining of the acantholytic cells as well as the adjacent dysplastic cells of the 2 specimens was assessed and compared with the immunohistochemical expression profiles noted in conventional moderate epithelial dysplasia
(MED), moderately differentiated keratinizing squamous cell carcinoma (MDSCC), and ASCC. Staining was assessed semiquantitatively as negative (no staining), faintly positive, or strongly positive. "Faint positivity" describes patchy to focal cytoplasmic or membranous immunoexpression in the cells of interest, whereas "strong positivity" was used to describe diffuse, crisp staining of all cells under scrutiny.

\section{RESULTS}

The immunohistochemical staining results obtained are summarized in Table II. Evaluation of the initial biopsy of $\mathrm{AD}$ showed AE1/AE3 positivity that confirmed the epithelial nature of the acantholytic dysplastic cells located within the pseudoglandular spaces (Figure 4). Vimentin coexpression was also noted within these cells (Figure 5). The loss of membranous E-cadherin expression within the free-lying cells located within the pseudolumina contrasted with the strong membranous positivity of the adjacent keratinocytes (Figure 6). The loss of E-cadherin expression was accompanied by increased cytoplasmic expression of $\beta$-catenin (Figure

7). The immunoprofile of the second biopsy of verrucous carcinoma with adenoid structures was similar to the initial biopsy (Figure 8).

ASCC similarly showed loss of E-cadherin, vimentin coexpression, and cytoplasmic staining for $\beta$-catenin (Figures 9 and 10). In comparison, the keratinocytes in cases of MED and MDSCC lacked coexpression of vimentin, loss of E-cadherin, and cytoplasmic $\beta$-catenin expression.

\section{DISCUSSION}

Histologic variants of invasive squamous cell carci-noma within the upper aerodigestive tract, including spindle cell carcinoma, papillary 


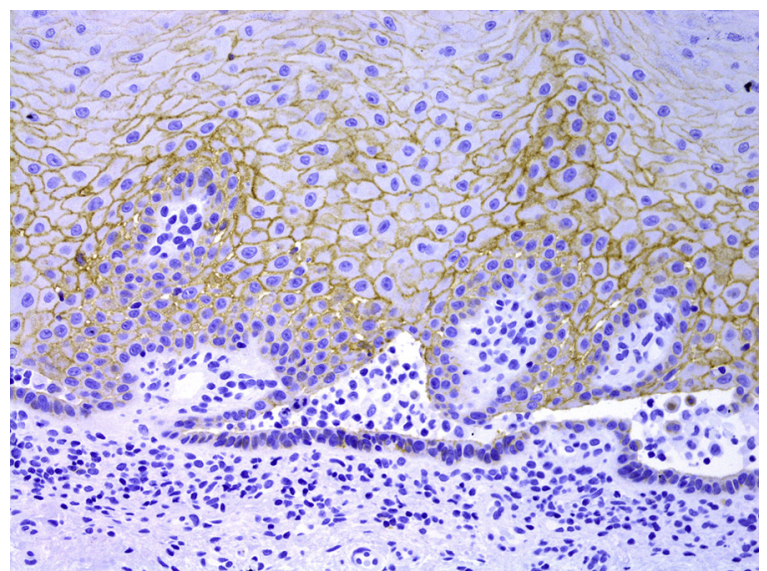

Fig. 6. Membranous E-cadherin expression is lost within the dysplastic acantholytic cells in the adenoid dysplasia biopsy, in contrast to the positive membrane staining that is evident in the surrounding epithelium (E-cadherin, original magnification $\times 400$ ). A high-resolution version of this slide is available as eSlide: VM00352.

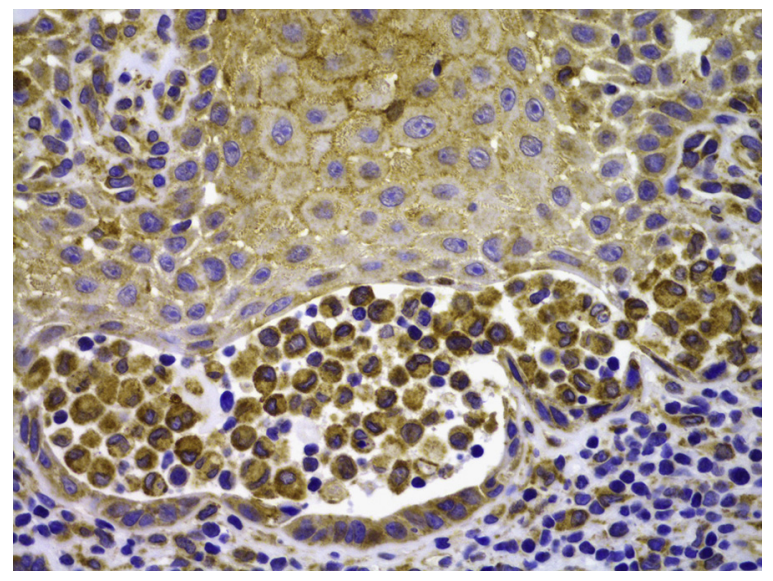

Fig. 7. Cytoplasmic expression of $\beta$-catenin within the dysplastic, acantholytic cells of the adenoid dysplasia biopsy

$(\beta$-catenin, original magnification $\times 200)$. A high-resolution version of this slide is available as eSlide: VM00353.

carcinoma, basaloid squamous cell carcinoma, and ASCC, are well docu-mented and have prognostic significance. ${ }^{5,6}$ This article documents the unique histomorphologic fea-tures in a case of oral epithelial dysplasia, which are reminiscent of those seen in ASCC. We have termed this unusual presentation adenoid dysplasia. The same unique features were subsequently noted in a second biopsy specimen obtained from the same patient 2 years later. A verrucous carcinoma was present in these tissue sections.

Lever ${ }^{7}$ first described a rare form of pseudoglandular squamous cell carcinoma on sun-exposed skin in 1947. This variant of SCC is variably termed adenoid SCC, pseudoglandular SCC, or angiosarcomatous SCC. Most lesions with this histomorphology are cutaneous and related to ultraviolet light exposure. Very few lesions have been described at mucosal sites within the upper aerodigestive tract. The first documented oral mucosal ASCC that involved the maxillary gingiva was reported by Goldman et al. in $1977 .{ }^{8}$ Several oral ASCCs have been identified at other sites, including the buccal mucosa, floor of mouth, and tongue. ${ }^{9,10}$ These lesions are reported to have a poorer prognosis than conventional SCC at the same site. ${ }^{5,9,11}$ Most of our accumulated knowledge in this regard is based on a handful of case reports with little follow-up information with which to reach definitive conclusions regarding the biologic behavior. Although the lesions in this patient shared overlapping histomorphology with those of ASCC, the presence of adenoid structures containing acantholytic epithelial cells within epithelial dysplasia or even in verrucous carcinoma has not been previously reported and was distinct enough to warrant documentation.

The shared features in $\mathrm{AD}$ and ASCC prompted examination of the immunoprofile of these 2 groups of lesions in comparison with that of conventional epithelial dysplasia and squamous cell carcinoma. The immuno-profile of ASCC signifies a phenotypic transition from epithelial to mesenchymal within the acantholytic cells, features that are associated with increased propensity for tumor progression and dissemination. Furthermore, this may be significant in the development of chemo-resistance. ${ }^{12}$ The immunoprofile may be useful in explaining the more aggressive clinical behavior attrib-uted to cases of ASCC. ASCC is associated with marked reduction in Ecadherin membrane positivity combined with higher levels of cytoplasmic $\beta$-catenin staining. In addition, there is coexpression of the mesenchymal marker vimentin, and this constellation of features is in keeping with epithelial-mesenchymal transition (EMT) ${ }^{13}$ The immunohistochemical expression levels of markers of EMT appear to be interdependent in the process of carcinogenesis that involves dysregulation of the Wnt signaling pathway. ${ }^{13}$ The acquisition of an EMT phenotype is strongly associated with increased potential for invasive tumor behavior as well as distant metastasis. The upregulation of EMT markers in conventional squamous cell carcinoma signifies a poor outcome and is particu-larly notable at the invasive tumor front However, these features are evident throughout the tumor in cases of ASCC. ${ }^{6,14,15}$ A recent study has found that increased vimentin expression within lesions of oral epithelial dysplasia closely correlates with histologic grade. This may represent a useful adjunct for the prediction of ma-lignant potential in high-risk lesions. ${ }^{16}$

E-cadherin is an essential cell adhesion molecule that maintains the structural integrity of epithelial cells as well as the cohesion between adjacent cells. The interaction 

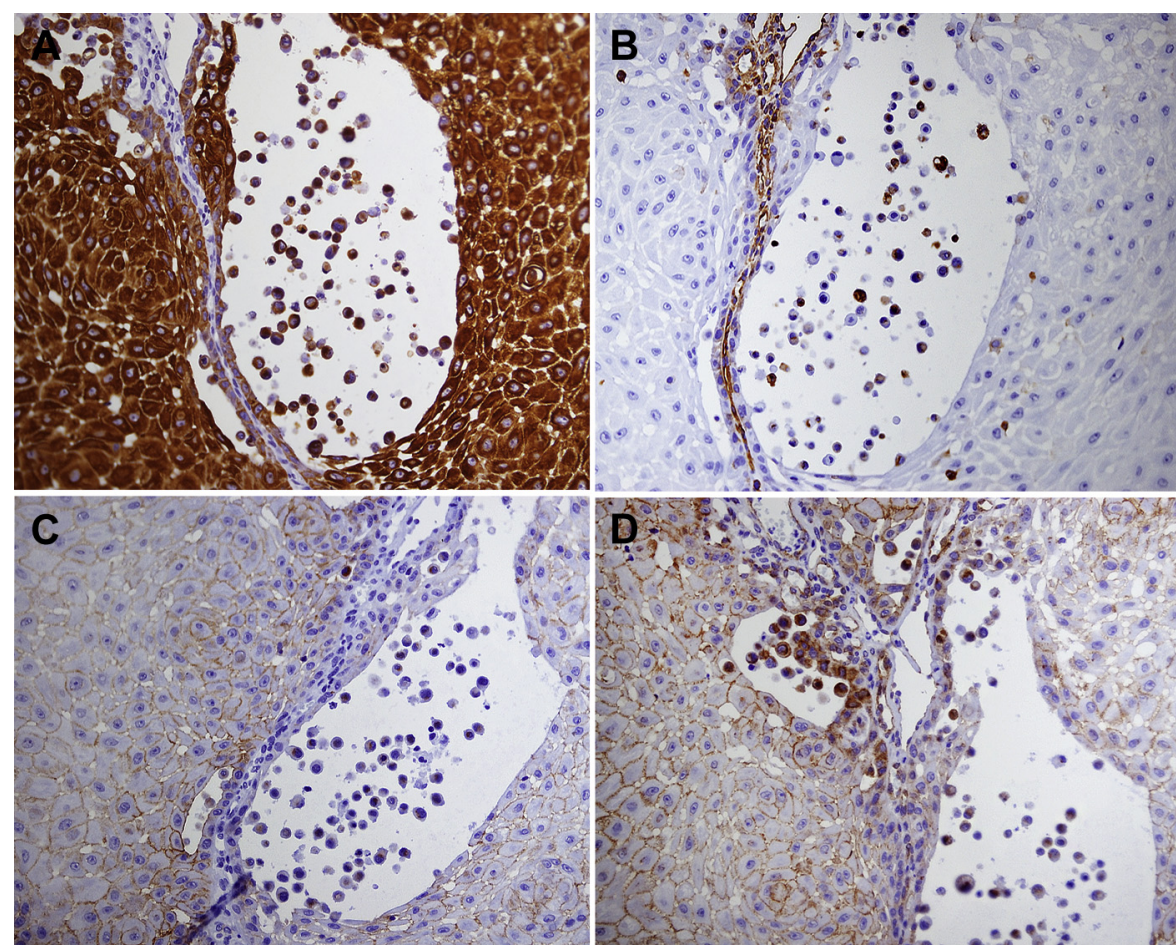

Fig. 8. Immunoprofile of the acantholytic cells in the pseudoglandular spaces in the verrucous carcinoma was similar to that of the adenoid dysplasia. A, AE1/AE3, original magnification $\times 200$. B, Vimentin, original magnification $\times 200$. C, E-cadherin, original magnification $\times 200$. D, $\beta$-catenin, original magnification $\times 200$. High-resolution versions of the slide images are available as eSlide: VM00355, eSlide: VM00357, eSlide: VM00356, eSlide: VM00354.

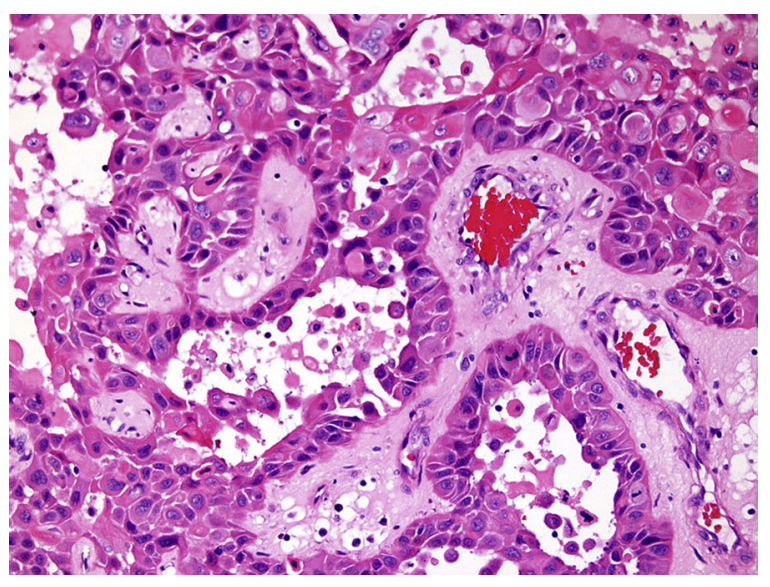

Fig. 9. The histomorphology of the acantholytic squamous cell carcinoma, where the invasive tumor contains pseudoglandular spaces in which free-floating malignant, dyskeratotic epithelial cells are identified (hematoxylin-eosin, original magnification $\times 400$ ). A high-resolution version of this slide is available as eSlide: VM00358.

with cytoplasmic catenins assists in linking E-cadherin to the actin cytoskeleton. ${ }^{13,17}$ E-cadherin and B-catenin form an interdependent cellular adhesion network that is highlighted by positive membranous immunoreactivity in normal oral epithelial cells and in cohesive tumor foci. ${ }^{13,14}$ The loss of membranous E-cadherin and $\beta$ catenin expression is associated with cytoplasmic $\beta$ catenin accumulation. Further increases in cytosolic $\beta$ catenin may result in nuclear translocation, where it functions as a transcription factor that plays a role in upregulation of genes involved in tumor invasion. ${ }^{13,14}$ The combination of downregulation of E-cadherin, aberrant cytoplasmic expression of $\beta$-catenin, and coexpression of vimentin thus signifies EMT and is associated with greater risk for malignant transformation in dysplastic lesions and more aggressive behavior in invasive lesions. ${ }^{6,13}$ The resultant loss of cell adhesion and the acquisition of cellular motility is an essential event in tumor cell invasion and distant spread. ${ }^{9}$

The acantholytic dysplastic cells in AD presented here show a loss of E-cadherin expression and increased cytoplasmic expression of $\beta$-catenin. These features recapitulate those seen in the cases of ASCC. The acantholytic cells within the second biopsy specimen showed similar immunohistochemical features. The features are in keeping with an EMT phenotype. The prognostic significance of these findings, particularly in the context of PVL, remains to be determined.

$\mathrm{AD}$ must be distinguished from conventional forms of oral epithelial dysplasia in which features of inflammatory-mediated spongiosis have occurred. In 


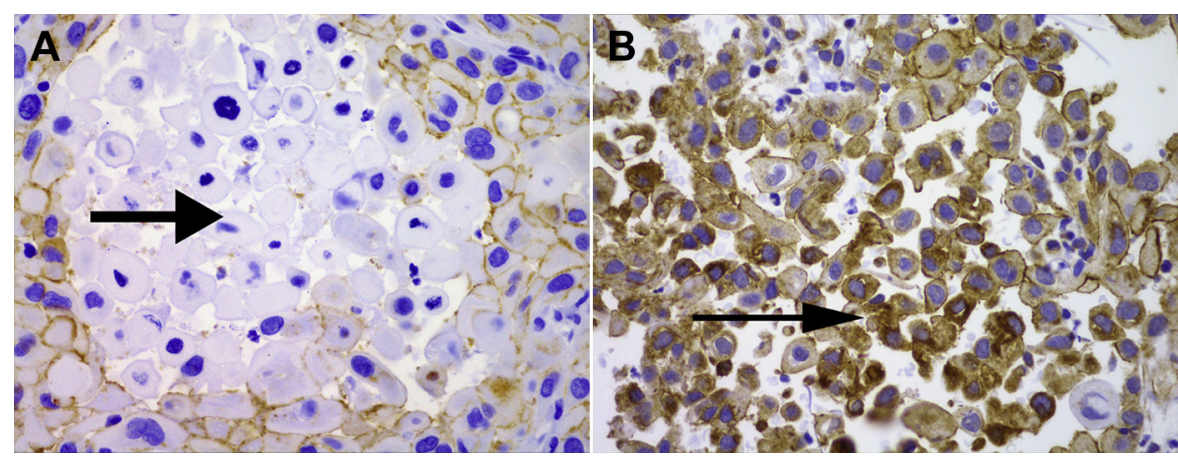

Fig. 10. A, Loss of E-cadherin expression in the free-floating tumor cells in acantholytic squamous cell carcinoma (E-cadherin, original magnification $\times 400$ ). B, Concomitant upregulation of cytoplasmic $\beta$-catenin expression in acantholytic squamous cell carcinoma $(\beta$-catenin, original magnification $\times 400)$. High-resolution versions of the slide images are available as eSlide: VM00359, eSlide: VM00360.

cases of spongiosis, the presence of intraepithelial inflammatory cells is accompanied by fluid ingress with resultant formation of intercellular spaces. There is, however, no true loss of intercellular bridges in these cases, and E-cadherin expression should remain unaltered. Tangential sectioning of cases of oral epithelial dysplasia may erroneously create the impression of AD, particularly in cases of marked interface mucositis and with psoriasiform hyperplasia. Serial sections of tissue should always be performed to exclude this possibility. Furthermore, derivation from a true invasive ASCC in small biopsy fragments should be distinguished from the purely intraepithelial form of $\mathrm{AD}$ described here. The consistent presence of these adenoid structures in multiple serial sections obtained from both the initial and second biopsy specimens precludes the possibility of a processing artifact. This is further substantiated by the focal presence of a lining of basal cuboidal epithelial cells in areas that accentuate the pseudoglandular to adenoid nature of the spaces noted.

Despite the histomorphologic similarities between the initial and subsequent biopsy specimens and ASCC, any comment on the biologic behavior remains purely speculative. Whether this case represents a most unusual morphologic variation or not and whether any prognostic significance can be attributed to these findings are questions that remain to be answered in the future. The aim here was to describe this form of dysplasia for the first time.

\section{REFERENCES}

1. Dionne KR, Warnakulasuriya S, Binti Zain R, Cheong SC. Potentially malignant disorders of the oral cavity: current practice and future directions in the clinic and laboratory [published online February 11, 2014]. Int J Cancer. http://dx.doi.org/10.1002/ijc. 28754.

2. Dost F, Le Cao K, Ford PJ, Ades C, Farah CS. Malignant transformation of oral epithelial dysplasia: a real-world evaluation of histopathologic grading. Oral Surg Oral Med Oral Pathol Oral Radiol. 2014;117:343-352.

3. van der Waal I. Potentially malignant disorders of the oral and oropharyngeal mucosa: terminology, classification and present concepts of management. Oral Oncol. 2009;45:317-323.

4. Kujan O, Oliver RJ, Khattab A, Roberts SA, Thakker N, Sloan P. Evaluation of a new binary system of grading oral epithelial dysplasia for prediction of malignant transformation. Oral Oncol. 2006;42:987-993.

5. Kerawala CJ. Acantholytic squamous cell carcinoma of the oral cavity: a more aggressive entity? Br J Oral Maxillofac Surg. 2009;47:123-125.

6. Gu X, Jiang R, Fowler MR. Acantholytic squamous cell carcinoma in upper aerodigestive tract: histopathology, immunohistochemical profile and epithelial mesenchymal transition phenotype change. Head Neck Pathol. 2012;6:438-444.

7. Lever WF. Adenoacanthoma of sweat gland-carcinoma of sweat glands with glandular and epidermal elements-report of four cases. Arch Derm Syphilol. 1947;56:157-171.

8. Goldman RL, Klein HZ, Sung M. Adenoid squamous cell carcinoma of the oral cavity: report of the first case arising in the tongue. Arch Otolaryngol. 1977;103:496-498.

9. Rosado P, Lequerica-Fernandez P, Fernandez S, Allonca E, Villallain L, de Vicente JC. E-cadherin and beta-catenin expression in well-differentiated and moderately-differentiated oral squamous cell carcinoma: relations with clinical variables. $\mathrm{Br} J$ Oral Maxillofac Surg. 2013;51:149-156.

10. Papadopoulou E, Tosios KI, Nikitakis N, Papadogeorgakis N, Sklavounou-Andrikopoulou A. Acantholytic squamous cell carcinoma of the gingiva: report of a case and review of the literature. Oral Surg Oral Med Oral Pathol Oral Radiol Endod. 2010;109:e67-e71.

11. Yeoh MS, Kim DD, Ghali GE. Acantholytic squamous cell carcinoma of the buccal mucosa: report of a case. J Oral Maxillofac Surg. 2012;70:1733-1738.

12. Kim H, Yoo SB, Sun P, et al. Alteration of the e-cadherin/betacatenin complex is an independent poor prognostic factor in lung adenocarcinoma. Korean J Pathol. 2013;47:44-51.

13. Chaw SY, Majeed AA, Dalley AJ, Chan A, Stein S, Farah CS Epithelial to mesenchymal transition (EMT) biomarkers-Ecadherin, beta-catenin, APC and Vimentin-in oral squamous cell carcinogenesis and transformation. Oral Oncol. 2012;48:9971006.

14. Liu LK, Jiang XY, Zhou XX, Wang DM, Song XL, Jiang HB. Upregulation of vimentin and aberrant expression of E-cadherin/ 
beta-catenin complex in oral squamous cell carcinomas: correlation with the clinicopathological features and patient outcome. Mod Pathol. 2010;23:213-224.

15. Mandal M, Myers JN, Lippman SM, et al. Epithelial to mesenchymal transition in head and neck squamous carcinoma: association of Src activation with E-cadherin down-regulation, vimentin expression, and aggressive tumor features. Cancer. 2008;112:2088-2100

16. Sawant S, Vaidya M, Chaukar D, et al. Clinical significance of aberrant vimentin expression in oral premalignant lesions and carcinomas. Oral Dis. 2014;20:453-465.
17. Thomas GJ, Speight PM. Cell adhesion molecules and oral cancer. Crit Rev Oral Biol Med. 2001;12:479-498. 